\title{
The Physics of Extra Dimensions
}

\author{
I. Antoniadis ${ }^{1}$ \\ Department of Physics, CERN - Theory Division, 1211 Geneva 23, Switzerland \\ ignatios.antoniadis@cern. $\mathrm{ch}^{\star \star}$
}

Lowering the string scale in the $\mathrm{TeV}$ region provides a theoretical framework for solving the mass hierarchy problem and unifying all interactions. The apparent weakness of gravity can then be accounted by the existence of large internal dimensions, in the submillimeter region, and transverse to a braneworld where our universe must be confined. I review the main properties of this scenario and its implications for observations at both particle colliders, and in non-accelerator gravity experiments. Such effects are for instance the production of Kaluza-Klein resonances, graviton emission in the bulk of extra dimensions, and a radical change of gravitational forces in the submillimeter range. I also discuss the warped case and localization of gravity in the presence of infinite size extra dimensions.

\section{Introduction}

During the last few decades, physics beyond the Standard Model (SM) was guided from the problem of mass hierarchy. This can be formulated as the question of why gravity appears to us so weak compared to the other three known fundamental interactions corresponding to the electromagnetic, weak and strong nuclear forces. Indeed, gravitational interactions are suppressed by a very high energy scale, the Planck mass $M_{P} \sim 10^{19} \mathrm{GeV}$, associated to a length $l_{P} \sim 10^{-35} \mathrm{~m}$, where they are expected to become important. In a quantum theory, the hierarchy implies a severe fine tuning of the fundamental parameters in more than 30 decimal places in order to keep the masses of elementary particles at their observed values. The reason is that quantum radiative corrections to all masses generated by the Higgs vacuum expectation value (VEV) are proportional to the ultraviolet cutoff which in the presence

${ }^{\star \star}$ On leave from CPHT (UMR CNRS 7644) Ecole Polytechnique, F-91128 Palaiseau 
of gravity is fixed by the Planck mass. As a result, all masses are "attracted" to become about $10^{16}$ times heavier than their observed values.

Besides compositeness, there are three main theories that have been proposed and studied extensively during the last years, corresponding to different approaches of dealing with the mass hierarchy problem. (1) Low energy supersymmetry with all superparticle masses in the $\mathrm{TeV}$ region. Indeed, in the limit of exact supersymmetry, quadratically divergent corrections to the Higgs self-energy are exactly cancelled, while in the softly broken case, they are cutoff by the supersymmetry breaking mass splittings. (2) TeV scale strings, in which quadratic divergences are cutoff by the string scale and low energy supersymmetry is not needed. (3) Split supersymmetry, where scalar masses are heavy while fermions (gauginos and higgsinos) are light. Thus, gauge coupling unification and dark matter candidate are preserved but the mass hierarchy should be stabilized by a different way and the low energy world appears to be fine-tuned. All these ideas are experimentally testable at high-energy particle colliders and in particular at LHC. Below, I discuss their implementation in string theory.

The appropriate and most convenient framework for low energy supersymmetry and grand unification is the perturbative heterotic string. Indeed, in this theory, gravity and gauge interactions have the same origin, as massless modes of the closed heterotic string, and they are unified at the string scale $M_{s}$. As a result, the Planck mass $M_{P}$ is predicted to be proportional to $M_{s}$ :

$$
M_{P}=M_{s} / g,
$$

where $g$ is the gauge coupling. In the simplest constructions all gauge couplings are the same at the string scale, given by the four-dimensional (4d) string coupling, and thus no grand unified group is needed for unification. In our conventions $\alpha_{\mathrm{GUT}}=g^{2} \simeq 0.04$, leading to a discrepancy between the string and grand unification scale $M_{\mathrm{GUT}}$ by almost two orders of magnitude. Explaining this gap introduces in general new parameters or a new scale, and the predictive power is essentially lost. This is the main defect of this framework, which remains though an open and interesting possibility.

The other two ideas have both as natural framework of realization type I string theory with D-branes. Unlike in the heterotic string, gauge and gravitational interactions have now different origin. The latter are described again by closed strings, while the former emerge as excitations of open strings with endpoints confined on D-branes [1. This leads to a braneworld description of our universe, which should be localized on a hypersurface, i.e. a membrane extended in $p$ spatial dimensions, called $p$-brane (see Fig. 1). Closed strings propagate in all nine dimensions of string theory: in those extended along the $p$-brane, called parallel, as well as in the transverse ones. On the contrary, open strings are attached on the $p$-brane. Obviously, our $p$-brane world must have at least the three known dimensions of space. But it may contain more: the extra $d_{\|}=p-3$ parallel dimensions must have a finite size, in order to be unobservable at present energies, and can be as large as $\mathrm{TeV}^{-1} \sim 10^{-18}$ 


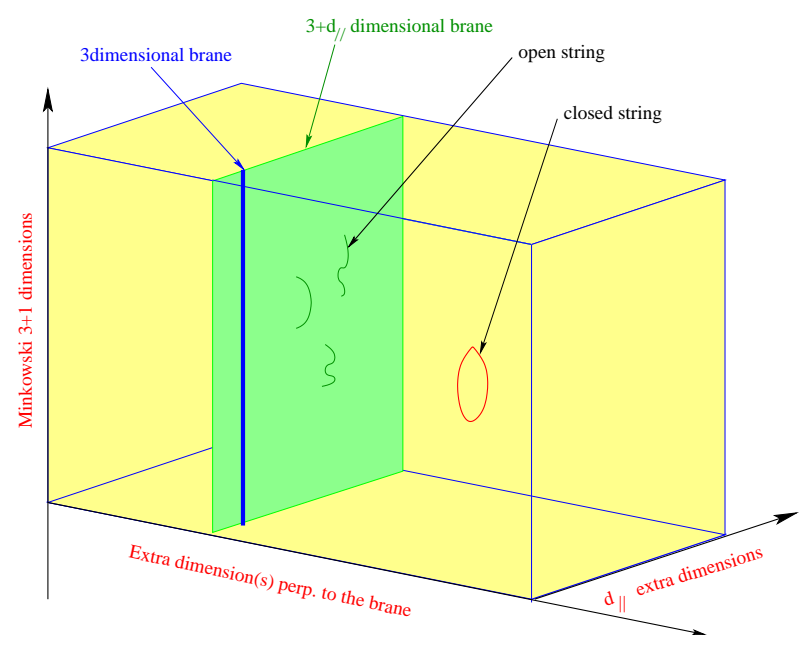

Fig. 1. In the type I string framework, our Universe contains, besides the three known spatial dimensions (denoted by a single blue line), some extra dimensions $\left(d_{\|}=p-3\right)$ parallel to our world $p$-brane (green plane) where endpoints of open strings are confined, as well as some transverse dimensions (yellow space) where only gravity described by closed strings can propagate.

$\mathrm{m}$ [2]. On the other hand, transverse dimensions interact with us only gravitationally and experimental bounds are much weaker: their size should be less than about $0.1 \mathrm{~mm}$ [3. In the following, I review the main properties and experimental signatures of low string scale models [4, 5].

\section{Framework}

In type I theory, the different origin of gauge and gravitational interactions implies that the relation between the Planck and string scales is not linear as (1) of the heterotic string. The requirement that string theory should be weakly coupled, constrain the size of all parallel dimensions to be of order of the string length, while transverse dimensions remain unrestricted. Assuming an isotropic transverse space of $n=9-p$ compact dimensions of common radius $R_{\perp}$, one finds:

$$
M_{P}^{2}=\frac{1}{g^{4}} M_{s}^{2+n} R_{\perp}^{n}, \quad g_{s} \simeq g^{2} .
$$

where $g_{s}$ is the string coupling. It follows that the type I string scale can be chosen hierarchically smaller than the Planck mass 6, 44 at the expense of introducing extra large transverse dimensions felt only by gravity, while 
keeping the string coupling small 4. The weakness of $4 \mathrm{~d}$ gravity compared to gauge interactions (ratio $M_{W} / M_{P}$ ) is then attributed to the largeness of the transverse space $R_{\perp}$ compared to the string length $l_{s}=M_{s}^{-1}$.

An important property of these models is that gravity becomes effectively $(4+n)$-dimensional with a strength comparable to those of gauge interactions at the string scale. The first relation of Eq. (2) can be understood as a consequence of the $(4+n)$-dimensional Gauss law for gravity, with

$$
M_{*}^{(4+n)}=M_{s}^{2+n} / g^{4}
$$

the effective scale of gravity in $4+n$ dimensions. Taking $M_{s} \simeq 1 \mathrm{TeV}$, one finds a size for the extra dimensions $R_{\perp}$ varying from $10^{8} \mathrm{~km}, .1 \mathrm{~mm}$, down to a Fermi for $n=1,2$, or 6 large dimensions, respectively. This shows that while $n=1$ is excluded, $n \geq 2$ is allowed by present experimental bounds on gravitational forces 3 , 7. Thus, in these models, gravity appears to us very weak at macroscopic scales because its intensity is spread in the "hidden" extra dimensions. At distances shorter than $R_{\perp}$, it should deviate from Newton's law, which may be possible to explore in laboratory experiments (see Fig. (2).

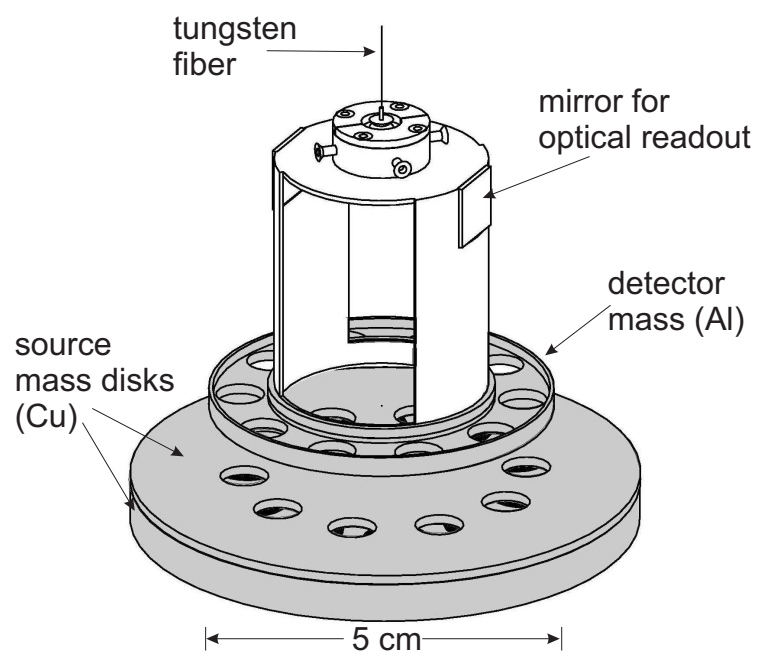

Fig. 2. Torsion pendulum that tested Newton's law at $130 \mathrm{~nm}$. Several sources of background noise were eliminated using appropriate devices.

The main experimental implications of $\mathrm{TeV}$ scale strings in particle accelerators are of three types, in correspondence with the three different sectors that are generally present: (i) new compactified parallel dimensions, (ii) new extra large transverse dimensions and low scale quantum gravity, and (iii) genuine string and quantum gravity effects. On the other hand, there exist 
interesting implications in non accelerator table-top experiments due to the exchange of gravitons or other possible states living in the bulk.

\section{Experimental implications in accelerators}

\subsection{World-brane extra dimensions}

In this case $R M_{s} \gtrsim 1$, and the associated compactification scale $R_{\|}^{-1}$ would be the first scale of new physics that should be found increasing the beam energy [2, 8. There are several reasons for the existence of such dimensions. It is a logical possibility, since out of the six extra dimensions of string theory only two are needed for lowering the string scale, and thus the effective $p$-brane of our world has in general $d_{\|} \equiv p-3 \leq 4$. Moreover, they can be used to address several physical problems in braneworld models, such as obtaining different SM gauge couplings, explaining fermion mass hierarchies due to different localization points of quarks and leptons in the extra dimensions, providing calculable mechanisms of supersymmetry breaking, etc.

The main consequence is the existence of Kaluza-Klein (KK) excitations for all SM particles that propagate along the extra parallel dimensions. Their masses are given by:

$$
M_{m}^{2}=M_{0}^{2}+\frac{m^{2}}{R_{\|}^{2}} \quad ; \quad m=0, \pm 1, \pm 2, \ldots
$$

where we used $d_{\|}=1$, and $M_{0}$ is the higher dimensional mass. The zero-mode $m=0$ is identified with the $4 \mathrm{~d}$ state, while the higher modes have the same quantum numbers with the lowest one, except for their mass given in (44). There are two types of experimental signatures of such dimensions [8, 9, 10]: (i) virtual exchange of KK excitations, leading to deviations in cross-sections compared to the SM prediction, that can be used to extract bounds on the compactification scale; (ii) direct production of KK modes.

On general grounds, there can be two different kinds of models with qualitatively different signatures depending on the localization properties of matter fermion fields. If the latter are localized in $3 \mathrm{~d}$ brane intersections, they do not have excitations and KK momentum is not conserved because of the breaking of translation invariance in the extra dimension(s). KK modes of gauge bosons are then singly produced giving rise to generally strong bounds on the compactification scale and new resonances that can be observed in experiments. Otherwise, they can be produced only in pairs due to the KK momentum conservation, making the bounds weaker but the resonances difficult to observe.

When the internal momentum is conserved, the interaction vertex involving KK modes has the same 4d tree-level gauge coupling. On the other hand, their couplings to localized matter have an exponential form factor suppressing the interactions of heavy modes. This form factor can be viewed as the 
fact that the branes intersection has a finite thickness. For instance, the coupling of the KK excitations of gauge fields $A^{\mu}(x, y)=\sum_{m} A_{m}^{\mu} \exp i \frac{m y}{R_{\|}}$to the charge density $j_{\mu}(x)$ of massless localized fermions is described by the effective action [11]:

$$
\int d^{4} x \sum_{m} e^{-\ln 16 \frac{m^{2} l_{s}^{2}}{2 R_{\|}^{2}}} j_{\mu}(x) A_{m}^{\mu}(x) .
$$

After Fourier transform in position space, it becomes:

$$
\int d^{4} x d y \frac{1}{(2 \pi \ln 16)^{2}} e^{-\frac{y^{2} M_{s}^{2}}{2 \ln 16}} j_{\mu}(x) A^{\mu}(x, y),
$$

from which we see that localized fermions form a Gaussian distribution of charge with a width $\sigma=\sqrt{\ln 16} l_{s} \sim 1.66 l_{s}$.

To simplify the analysis, let us consider first the case $d_{\|}=1$ where some of the gauge fields arise from an effective 4-brane, while fermions are localized states on brane intersections. Since the corresponding gauge couplings are reduced by the size of the large dimension $R_{\|} M_{s}$ compared to the others, one can account for the ratio of the weak to strong interactions strengths if the $S U(2)$ brane extends along the extra dimension, while $S U(3)$ does not. As a result, there are 3 distinct cases to study [10, denoted by $(t, l, l),(t, l, t)$ and $(t, t, l)$, where the three positions in the brackets correspond to the three SM gauge group factors $S U(3) \times S U(2) \times U(1)$ and those with $l$ (longitudinal) feel the extra dimension, while those with $t$ (transverse) do not.

In the $(t, l, l)$ case, there are KK excitations of $S U(2) \times U(1)$ gauge bosons: $W_{ \pm}^{(m)}, \gamma^{(m)}$ and $Z^{(m)}$. Performing a $\chi^{2}$ fit of the electroweak observables, one finds that if the Higgs is a bulk state $(l), R_{\|}^{-1} \gtrsim 3.5 \mathrm{TeV}[12$. This implies that LHC can produce at most the first KK mode. Different choices for localization of matter and Higgs fields lead to bounds, lying in the range $1-5 \mathrm{TeV}$ 12.

In addition to virtual effects, KK excitations can be produced on-shell at LHC as new resonances 9] (see Fig. 31). There are two different channels, neutral Drell-Yan processes $p p \rightarrow l^{+} l^{-} X$ and the charged channel $l^{ \pm} \nu$, corresponding to the production of the KK modes $\gamma^{(1)}, Z^{(1)}$ and $W_{ \pm}^{(1)}$, respectively. The discovery limits are about $6 \mathrm{TeV}$, while the exclusion bounds $15 \mathrm{TeV}$. An interesting observation in the case of $\gamma^{(1)}+Z^{(1)}$ is that interferences can lead to a "dip" just before the resonance. There are some ways to distinguish the corresponding signals from other possible origin of new physics, such as models with new gauge bosons. In fact, in the $(t, l, l)$ and $(t, l, t)$ cases, one expects two resonances located practically at the same mass value. This property is not shared by most of other new gauge boson models. Moreover, the heights and widths of the resonances are directly related to those of SM gauge bosons in the corresponding channels.

In the $(t, l, t)$ case, only the $S U(2)$ factor feels the extra dimension and the limits set by the KK states of $W^{ \pm}$remain the same. On the other hand, in the $(t, t, l)$ case where only $U(1)_{Y}$ feels the extra dimension, the limits are 


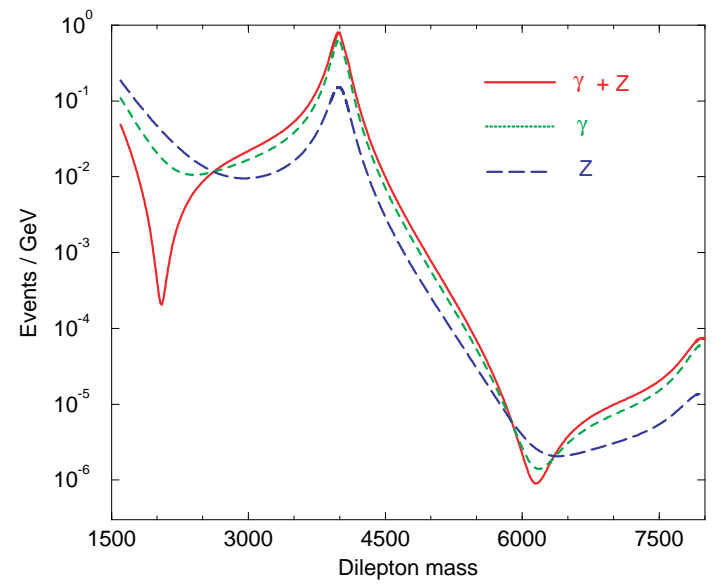

Fig. 3. Production of the first KK modes of the photon and of the $Z$ boson at LHC, decaying to electron-positron pairs. The number of expected events is plotted as a function of the energy of the pair in GeV. From highest to lowest: excitation of $\gamma+Z, \gamma$ and $Z$.

weaker and the exclusion bound is around $8 \mathrm{TeV}$. In addition to these simple possibilities, brane constructions lead often to cases where part of $U(1)_{Y}$ is $t$ and part is $l$. If $S U(2)$ is $l$ the limits come again from $W^{ \pm}$, while if it is $t$ then it will be difficult to distinguish this case from a generic extra $U(1)^{\prime}$. A good statistics would be needed to see the deviation in the tail of the resonance as being due to effects additional to those of a generic $U(1)^{\prime}$ resonance. Finally, in the case of two or more parallel dimensions, the sum in the exchange of the KK modes diverges in the limit $R_{\|} M_{s}>>1$ and needs to be regularized using the form factor (5). Cross-sections become bigger yielding stronger bounds, while resonances are closer implying that more of them could be reached by LHC.

On the other hand, if all SM particles propagate in the extra dimension (called universal) ${ }^{3}$, KK modes can only be produced in pairs and the lower bound on the compactification scale becomes weaker, of order of 300-500 GeV. Moreover, no resonances can be observed at LHC, so that this scenario appears very similar to low energy supersymmetry. In fact, KK parity can even play the role of R-parity, implying that the lightest KK mode is stable and can be a dark matter candidate in analogy to the LSP [13].

\footnotetext{
${ }^{3}$ Although interesting, this scenario seems difficult to be realized, since $4 \mathrm{~d}$ chirality requires non-trivial action of orbifold twists with localized chiral states at the fixed points.
} 
Table 1. Limits on $R_{\perp}$ in $\mathrm{mm}$.

\begin{tabular}{|c|c|c|c|}
\hline & & & \\
Experiment & $n=2$ & $n=4$ & $n=6$ \\
\hline \hline \multicolumn{4}{|c|}{ Collider bounds } \\
\hline LEP 2 & $5 \times 10^{-1}$ & $2 \times 10^{-8}$ & $7 \times 10^{-11}$ \\
\hline Tevatron & $5 \times 10^{-1}$ & $10^{-8}$ & $4 \times 10^{-11}$ \\
\hline LHC & $4 \times 10^{-3}$ & $6 \times 10^{-10}$ & $3 \times 10^{-12}$ \\
\hline NLC & $10^{-2}$ & $10^{-9}$ & $6 \times 10^{-12}$ \\
\hline \hline \multicolumn{4}{|c|}{ Present non-collider bounds } \\
\hline SN1987A & $3 \times 10^{-4}$ & $10^{-8}$ & $6 \times 10^{-10}$ \\
\hline COMPTEL & $5 \times 10^{-5}$ & - & - \\
\hline
\end{tabular}

\subsection{Extra large transverse dimensions}

The main experimental signal is gravitational radiation in the bulk from any physical process on the world-brane. In fact, the very existence of branes breaks translation invariance in the transverse dimensions and gravitons can be emitted from the brane into the bulk. During a collision of center of mass energy $\sqrt{s}$, there are $\sim\left(\sqrt{s} R_{\perp}\right)^{n}$ KK excitations of gravitons with tiny masses, that can be emitted. Each of these states looks from the $4 \mathrm{~d}$ point of view as a massive, quasi-stable, extremely weakly coupled $\left(s / M_{P}^{2}\right.$ suppressed) particle that escapes from the detector. The total effect is a missing-energy crosssection roughly of order:

$$
\frac{\left(\sqrt{s} R_{\perp}\right)^{n}}{M_{P}^{2}} \sim \frac{1}{s}\left(\frac{\sqrt{s}}{M_{s}}\right)^{n+2}
$$

Explicit computation of these effects leads to the bounds given in Table 1 However, larger radii are allowed if one relaxes the assumption of isotropy, by taking for instance two large dimensions with different radii.

Fig. 4 shows the cross-section for graviton emission in the bulk, corresponding to the process $p p \rightarrow$ jet + graviton at LHC, together with the SM background [14. For a given value of $M_{s}$, the cross-section for graviton emission decreases with the number of large transverse dimensions, in contrast to the case of parallel dimensions. The reason is that gravity becomes weaker if there are more dimensions because there is more space for the gravitational field to escape. There is a particular energy and angular distribution of the produced gravitons that arise from the distribution in mass of KK states of spin-2. This can be contrasted to other sources of missing energy and might be a smoking gun for the extra dimensional nature of such a signal.

In Table 1] there are also included astrophysical and cosmological bounds. Astrophysical bounds [15, 16] arise from the requirement that the radiation of gravitons should not carry on too much of the gravitational binding energy released during core collapse of supernovae. In fact, the measurements of 


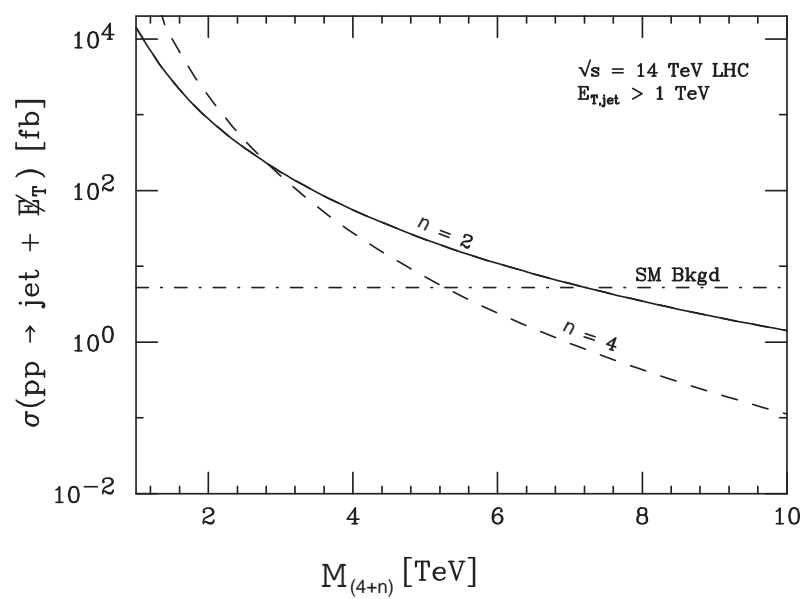

Fig. 4. Missing energy due to graviton emission at LHC, as a function of the higherdimensional gravity scale $M_{*}$, produced together with a hadronic jet. The expected cross-section is shown for $n=2$ and $n=4$ extra dimensions, together with the SM background.

Kamiokande and IMB for SN1987A suggest that the main channel is neutrino fluxes. The best cosmological bound [17] is obtained from requiring that decay of bulk gravitons to photons do not generate a spike in the energy spectrum of the photon background measured by the COMPTEL instrument. Bulk gravitons are expected to be produced just before nucleosynthesis due to thermal radiation from the brane. The limits assume that the temperature was at most $1 \mathrm{MeV}$ as nucleosynthesis begins, and become stronger if temperature is increased.

\subsection{String effects}

At low energies, the interaction of light (string) states is described by an effective field theory. Their exchange generates in particular four-fermion operators that can be used to extract independent bounds on the string scale. In analogy with the bounds on longitudinal extra dimensions, there are two cases depending on the localization properties of matter fermions. If they come from open strings with both ends on the same stack of branes, exchange of massive open string modes gives rise to dimension eight effective operators, involving four fermions and two space-time derivatives [18, 11. The corresponding bounds on the string scale are then around $500 \mathrm{GeV}$. On the other hand, if matter fermions are localized on non-trivial brane intersections, one obtains dimension six four-fermion operators and the bounds become stronger: $M_{s} \gtrsim 2-3$ $\mathrm{TeV}$ [11, 5]. At energies higher than the string scale, new spectacular phenomena are expected to occur, related to string physics and quantum gravity 
effects, such as possible micro-black hole production [19]. Particle accelerators would then become the best tools for studying quantum gravity and string theory.

\section{Supersymmetry in the bulk and short range forces}

\subsection{Sub-millimeter forces}

Besides the spectacular predictions in accelerators, there are also modifications of gravitation in the sub-millimeter range, which can be tested in "table-top" experiments that measure gravity at short distances. There are three categories of such predictions:

(i) Deviations from the Newton's law $1 / r^{2}$ behavior to $1 / r^{2+n}$, which can be observable for $n=2$ large transverse dimensions of sub-millimeter size. This case is particularly attractive on theoretical grounds because of the logarithmic sensitivity of SM couplings on the size of transverse space [20], that allows to determine the hierarchy [21].

(ii) New scalar forces in the sub-millimeter range, related to the mechanism of supersymmetry breaking, and mediated by light scalar fields $\varphi$ with masses [22, 4]:

$$
m_{\varphi} \simeq \frac{m_{\text {susy }}^{2}}{M_{P}} \simeq 10^{-4}-10^{-6} \mathrm{eV},
$$

for a supersymmetry breaking scale $m_{\text {susy }} \simeq 1-10 \mathrm{TeV}$. They correspond to Compton wavelengths of $1 \mathrm{~mm}$ to $10 \mu \mathrm{m}$. $m_{\text {susy }}$ can be either $1 / R_{\|}$if supersymmetry is broken by compactification [22], or the string scale if it is broken "maximally" on our world-brane 4. A universal attractive scalar force is mediated by the radion modulus $\varphi \equiv M_{P} \ln R$, with $R$ the radius of the longitudinal or transverse dimension(s). In the former case, the result (8) follows from the behavior of the vacuum energy density $\Lambda \sim 1 / R_{\|}^{4}$ for large $R_{\|}$(up to logarithmic corrections). In the latter, supersymmetry is broken primarily on the brane, and thus its transmission to the bulk is gravitationally suppressed, leading to (8). For $n=2$, there may be an enhancement factor of the radion mass by $\ln R_{\perp} M_{s} \simeq 30$ decreasing its wavelength by an order of magnitude 21].

The coupling of the radius modulus to matter relative to gravity can be easily computed and is given by:

$$
\sqrt{\alpha_{\varphi}}=\frac{1}{M} \frac{\partial M}{\partial \varphi} ; \quad \alpha_{\varphi}=\left\{\begin{array}{l}
\frac{\partial \ln \Lambda_{\mathrm{QCD}}}{\partial \ln R} \simeq \frac{1}{3} \text { for } R_{\|} \\
\frac{2 n}{n+2}=1-1.5 \text { for } R_{\perp}
\end{array}\right.
$$

where $M$ denotes a generic physical mass. In the longitudinal case, the coupling arises dominantly through the radius dependence of the QCD gauge coupling [22], while in the case of transverse dimension, it can be deduced from 
the rescaling of the metric which changes the string to the Einstein frame and depends slightly on the bulk dimensionality $(\alpha=1-1.5$ for $n=2-6)$ 21. Such a force can be tested in microgravity experiments and should be contrasted with the change of Newton's law due the presence of extra dimensions that is observable only for $n=2[\underline{3}, 7$. The resulting bounds from an analysis of the radion effects are $[3$ :

$$
M_{*} \gtrsim 3-4.5 \mathrm{TeV} \text { for } n=2-6 .
$$

In principle there can be other light moduli which couple with even larger strengths. For example the dilaton, whose VEV determines the string coupling, if it does not acquire large mass from some dynamical supersymmetric mechanism, can lead to a force of strength 2000 times bigger than gravity 23]. (iii) Non universal repulsive forces much stronger than gravity, mediated by possible abelian gauge fields in the bulk [15, 24]. Such fields acquire tiny masses of the order of $M_{s}^{2} / M_{P}$, as in (8), due to brane localized anomalies [24]. Although their gauge coupling is infinitesimally small, $g_{A} \sim M_{s} / M_{P} \simeq 10^{-16}$, it is still bigger that the gravitational coupling $E / M_{P}$ for typical energies $E \sim 1$ $\mathrm{GeV}$, and the strength of the new force would be $10^{6}-10^{8}$ stronger than gravity. This is an interesting region which will be soon explored in micro-gravity experiments (see Fig. 50. Note that in this case supernova constraints impose that there should be at least four large extra dimensions in the bulk [15].

In Fig. [5 we depict the actual information from previous, present and upcoming experiments [7, 21. The solid lines indicate the present limits from the experiments indicated. The excluded regions lie above these solid lines. Measuring gravitational strength forces at short distances is challenging. The dashed thick lines give the expected sensitivity of the various experiments, which will improve the actual limits by roughly two orders of magnitude, while the horizontal dashed lines correspond to the theoretical predictions for the graviton in the case $n=2$ and for the radion in the transverse case. These limits are compared to those obtained from particle accelerator experiments in Table 1 Finally, in Figs. [6 and 7 we display recent improved bounds for new forces at very short distances by focusing on the right hand side of Fig. 5 near the origin [7.

\subsection{Brane non-linear supersymmetry}

When the closed string sector is supersymmetric, supersymmetry on a generic brane configuration is non-linearly realized even if the spectrum is not supersymmetric and brane fields have no superpartners. The reason is that the gravitino must couple to a conserved current locally, implying the existence of a goldstino on the brane world-volume. The goldstino is exactly massless in the infinite (transverse) volume limit and is expected to acquire a small

mass suppressed by the volume, of order (8). In the standard realization, its coupling to matter is given via the energy momentum tensor [25], while in 


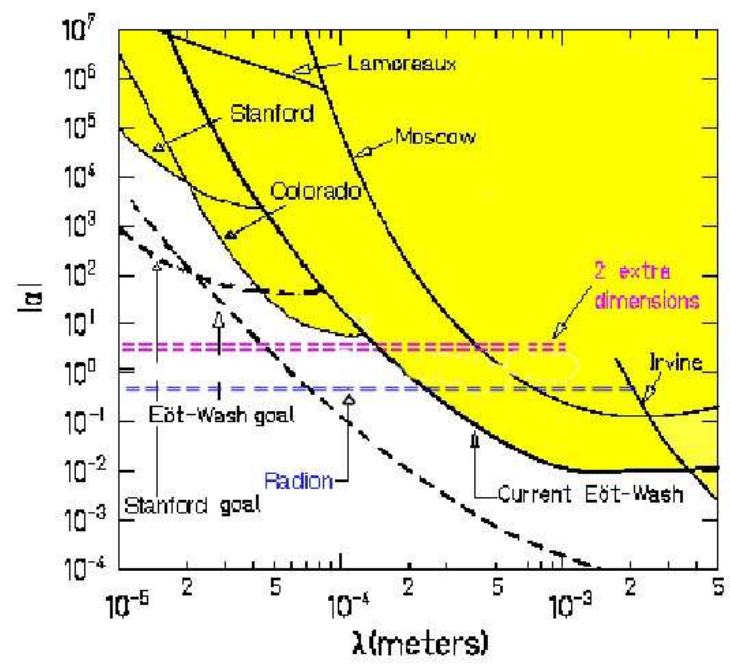

Fig. 5. Present limits on non-Newtonian forces at short distances (yellow regions), as a function of their range $\lambda$ and their strength relative to gravity $\alpha$. The limits are compared to new forces mediated by the graviton in the case of two large extra dimensions, and by the radion.

general there are more terms invariant under non-linear supersymmetry that have been classified, up to dimension eight [26, 27].

An explicit computation was performed for a generic intersection of two brane stacks, leading to three irreducible couplings, besides the standard one [27]: two of dimension six involving the goldstino, a matter fermion and a scalar or gauge field, and one four-fermion operator of dimension eight. Their strength is set by the goldstino decay constant $\kappa$, up to model-independent numerical coefficients which are independent of the brane angles. Obviously, at low energies the dominant operators are those of dimension six. In the minimal case of (non-supersymmetric) SM, only one of these two operators may exist, that couples the goldstino $\chi$ with the Higgs $H$ and a lepton doublet $L$ :

$$
\mathcal{L}_{\chi}^{\text {int }}=2 \kappa\left(D_{\mu} H\right)\left(L D^{\mu} \chi\right)+\text { h.c. }
$$

where the goldstino decay constant is given by the total brane tension

$$
\frac{1}{2 \kappa^{2}}=N_{1} T_{1}+N_{2} T_{2} ; \quad T_{i}=\frac{M_{s}^{4}}{4 \pi^{2} g_{i}^{2}},
$$

with $N_{i}$ the number of branes in each stack. It is important to notice that the effective interaction (11) conserves the total lepton number $L$, as long as we 


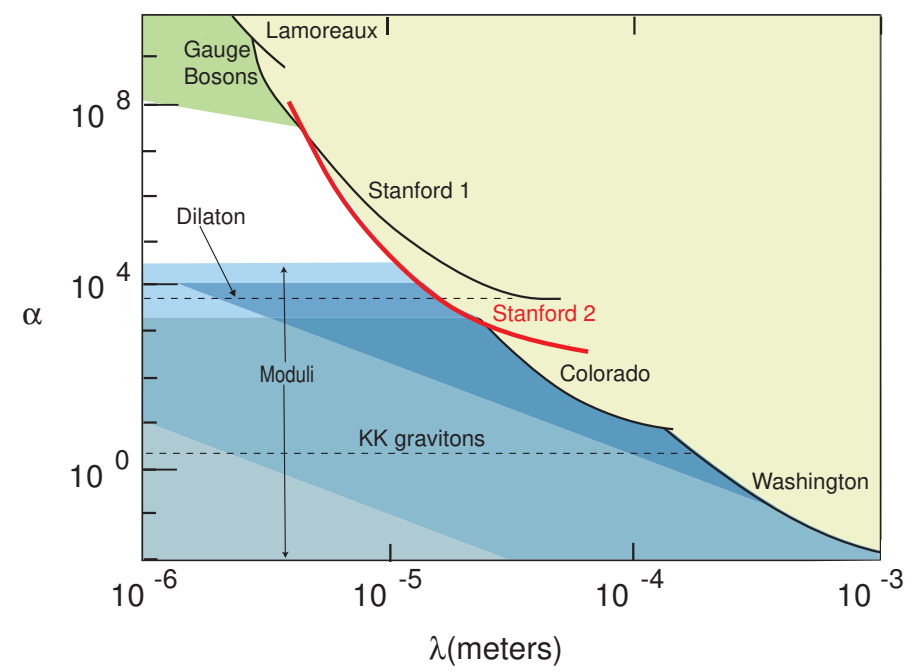

Fig. 6. Bounds on non-Newtonian forces in the range 6-20 $\mu \mathrm{m}$ (see S. J. Smullin et al. in Ref. [7]).

assign to the goldstino a total lepton number $L(\chi)=-1$ 28]. To simplify the

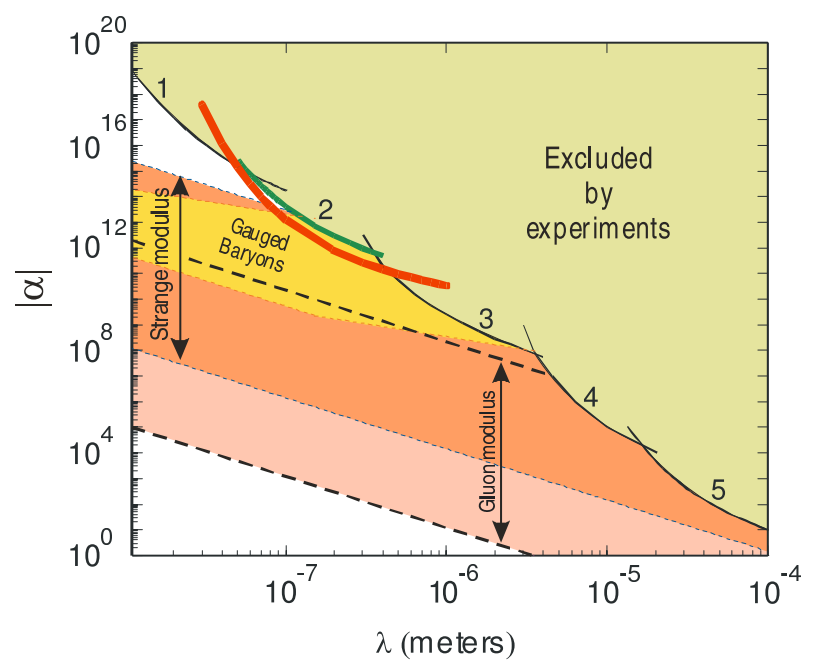

Fig. 7. Bounds on non-Newtonian forces in the range around $200 \mathrm{~nm}$ (see R. S. Decca et al. in Ref. [7). Curves 4 and 5 correspond to Stanford and Colorado experiments, respectively, of Fig. 6] (see also J C. Long and J. C. Price of Ref. 7]). 
analysis, we will consider the simplest case where (11) exists only for the first generation and $L$ is the electron doublet [28].

The effective interaction (11) gives rise mainly to the decays $W^{ \pm} \rightarrow e^{ \pm} \chi$ and $Z, H \rightarrow \nu \chi$. It turns out that the invisible $Z$ width gives the strongest limit on $\kappa$ which can be translated to a bound on the string scale $M_{s} \gtrsim$ $500 \mathrm{GeV}$, comparable to other collider bounds. This allows for the striking possibility of a Higgs boson decaying dominantly, or at least with a sizable branching ratio, via such an invisible mode, for a wide range of the parameter space $\left(M_{s}, m_{H}\right)$, as seen in Fig. 8
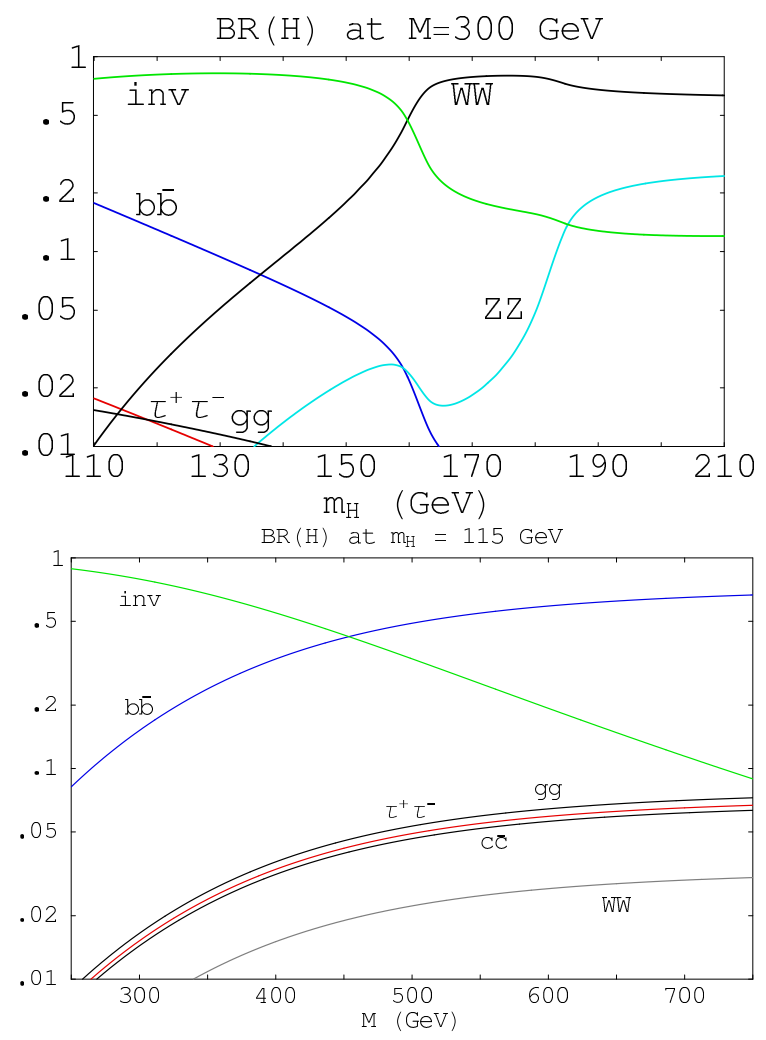

Fig. 8. Higgs branching rations, as functions either of the Higgs mass $m_{H}$ for a fixed value of the string scale $M_{s} \simeq 2 M=600 \mathrm{GeV}$, or of $M \simeq M_{s} / 2$ for $m_{H}=115$ $\mathrm{GeV}$. 


\section{Electroweak symmetry breaking}

Non-supersymmetric TeV strings offer also a framework to realize gauge symmetry breaking radiatively. Indeed, from the effective field theory point of view, one expects quadratically divergent one-loop contributions to the masses of scalar fields. The divergences are cut off by $M_{s}$ and if the corrections are negative, they can induce electroweak symmetry breaking and explain the mild hierarchy between the weak and a string scale at a few $\mathrm{TeV}$, in terms of a loop factor 29. More precisely, in the minimal case of one Higgs doublet $H$, the scalar potential is:

$$
V=\lambda\left(H^{\dagger} H\right)^{2}+\mu^{2}\left(H^{\dagger} H\right),
$$

where $\lambda$ arises at tree-level. Moreover, in any model where the Higgs field comes from an open string with both ends fixed on the same brane stack, it is given by an appropriate truncation of a supersymmetric theory. Within the minimal spectrum of the SM, $\lambda=\left(g_{2}^{2}+g^{\prime 2}\right) / 8$, with $g_{2}$ and $g^{\prime}$ the $S U(2)$ and $U(1)_{Y}$ gauge couplings. On the other hand, $\mu^{2}$ is generated at one loop:

$$
\mu^{2}=-\varepsilon^{2} g^{2} M_{s}^{2},
$$

where $\varepsilon$ is a loop factor that can be estimated from a toy model computation and varies in the region $\epsilon \sim 10^{-1}-10^{-3}$.

Indeed, consider for illustration a simple case where the whole one-loop effective potential of a scalar field can be computed. We assume for instance one extra dimension compactified on a circle of radius $R>1$ (in string units). An interesting situation is provided by a class of models where a non-vanishing VEV for a scalar (Higgs) field $\phi$ results in shifting the mass of each KK excitation by a constant $a(\phi)$ :

$$
M_{m}^{2}=\left(\frac{m+a(\phi)}{R}\right)^{2}
$$

with $m$ the KK integer momentum number. Such mass shifts arise for instance in the presence of a Wilson line, $a=q \oint \frac{d y}{2 \pi} g A$, where $A$ is the internal component of a gauge field with gauge coupling $g$, and $q$ is the charge of a given state under the corresponding generator. A straightforward computation shows that the $\phi$-dependent part of the one-loop effective potential is given by 30]:

$$
V_{\text {eff }}=-\operatorname{Tr}(-)^{F} \frac{R}{32 \pi^{3 / 2}} \sum_{n} e^{2 \pi i n a} \int_{0}^{\infty} d l l^{3 / 2} f_{s}(l) e^{-\pi^{2} n^{2} R^{2} l}
$$

where $F=0,1$ for bosons and fermions, respectively. We have included a regulating function $f_{s}(l)$ which contains for example the effects of string oscillators. To understand its role we will consider the two limits $R>>1$ and 
$R<<1$. In the first case only the $l \rightarrow 0$ region contributes to the integral. This means that the effective potential receives sizable contributions only from the infrared (field theory) degrees of freedom. In this limit we would have $f_{s}(l) \rightarrow 1$. For example, in the string model considered in [29]:

$$
f_{s}(l)=\left[\frac{1}{4 l} \frac{\theta_{2}}{\eta^{3}}\left(i l+\frac{1}{2}\right)\right]^{4} \rightarrow 1 \quad \text { for } \quad l \rightarrow 0,
$$

and the field theory result is finite and can be explicitly computed. As a result of the Taylor expansion around $a=0$, we are able to extract the oneloop contribution to the coefficient of the term of the potential quadratic in the Higgs field. It is given by a loop factor times the compactification scale 30. One thus obtains $\mu^{2} \sim g^{2} / R^{2}$ up to a proportionality constant which is calculable in the effective field theory. On the other hand, if we consider $R \rightarrow$ 0 , which by $T$-duality corresponds to taking the extra dimension as transverse and very large, the one-loop effective potential receives contributions from the whole tower of string oscillators as appearing in $f_{s}(l)$, leading to squared masses given by a loop factor times $M_{s}^{2}$, according to eq. (14).

More precisely, from the expression (16), one finds:

$$
\varepsilon^{2}(R)=\frac{1}{2 \pi^{2}} \int_{0}^{\infty} \frac{d l}{(2 l)^{5 / 2}} \frac{\theta_{2}^{4}}{4 \eta^{12}}\left(i l+\frac{1}{2}\right) R^{3} \sum_{n} n^{2} e^{-2 \pi n^{2} R^{2} l},
$$

which is plotted in Fig. 9 For the asymptotic value $R \rightarrow 0$ (corresponding

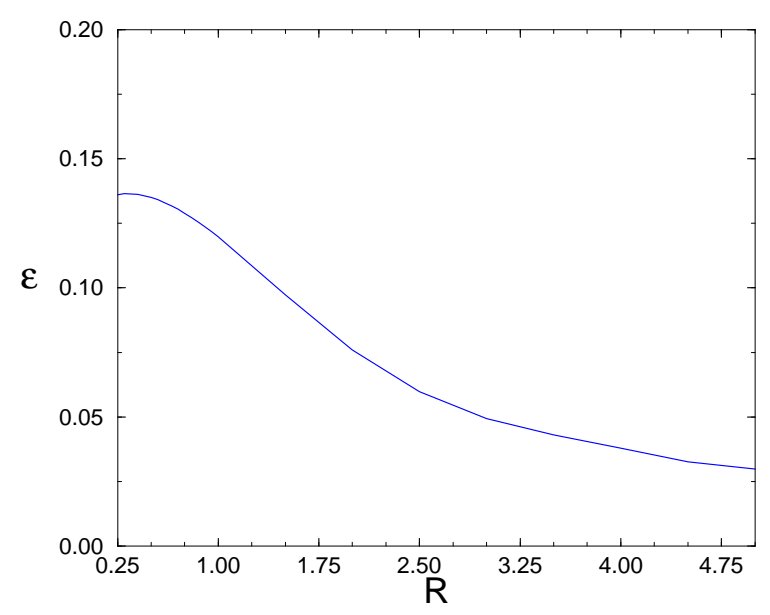

Fig. 9. The coefficient $\varepsilon$ of the one loop Higgs mass (14).

upon T-duality to a large transverse dimension of radius $1 / R), \varepsilon(0) \simeq 0.14$, 
and the effective cut-off for the mass term is $M_{s}$, as can be seen from Eq. (14). At large $R, \mu^{2}(R)$ falls off as $1 / R^{2}$, which is the effective cut-off in the limit $R \rightarrow \infty$, as we argued above, in agreement with field theory results in the presence of a compactified extra dimension 31 22. In fact, in the limit $R \rightarrow$ $\infty$, an analytic approximation to $\varepsilon(R)$ gives:

$$
\varepsilon(R) \simeq \frac{\varepsilon_{\infty}}{M_{s} R}, \quad \varepsilon_{\infty}^{2}=\frac{3 \zeta(5)}{4 \pi^{4}} \simeq 0.008 .
$$

The potential (13) has the usual minimum, given by the VEV of the neutral component of the Higgs doublet $v=\sqrt{-\mu^{2} / \lambda}$. Using the relation of $v$ with the $Z$ gauge boson mass, $M_{Z}^{2}=\left(g_{2}^{2}+g^{\prime 2}\right) v^{2} / 4$, and the expression of the quartic coupling $\lambda$, one obtains for the Higgs mass a prediction which is the MSSM value for $\tan \beta \rightarrow \infty$ and $m_{A} \rightarrow \infty: m_{H}=M_{Z}$. The tree level Higgs mass is known to receive important radiative corrections from the top-quark sector and rises to values around $120 \mathrm{GeV}$. Furthermore, from (14), one can compute $M_{s}$ in terms of the Higgs mass $m_{H}^{2}=-2 \mu^{2}$ :

$$
M_{s}=\frac{m_{H}}{\sqrt{2} g \varepsilon},
$$

yielding naturally values in the $\mathrm{TeV}$ range.

\section{Standard Model on D-branes}

The gauge group closest to the Standard Model one can easily obtain with D-branes is $U(3) \times U(2) \times U(1)$. The first factor arises from three coincident "color" D-branes. An open string with one end on them is a triplet under $S U(3)$ and carries the same $U(1)$ charge for all three components. Thus, the $U(1)$ factor of $U(3)$ has to be identified with gauged baryon number. Similarly, $U(2)$ arises from two coincident "weak" D-branes and the corresponding abelian factor is identified with gauged weak-doublet number. Finally, an extra $U(1)$ D-brane is necessary in order to accommodate the Standard Model without breaking the baryon number 32 . In principle this $U(1)$ brane can be chosen to be independent of the other two collections with its own gauge coupling. To improve the predictability of the model, we choose to put it on top of either the color or the weak D-branes 33. In either case, the model has two independent gauge couplings $g_{3}$ and $g_{2}$ corresponding, respectively, to the gauge groups $U(3)$ and $U(2)$. The $U(1)$ gauge coupling $g_{1}$ is equal to either $g_{3}$ or $g_{2}$.

Let us denote by $Q_{3}, Q_{2}$ and $Q_{1}$ the three $U(1)$ charges of $U(3) \times U(2) \times$ $U(1)$, in a self explanatory notation. Under $S U(3) \times S U(2) \times U(1)_{3} \times U(1)_{2} \times$ $U(1)_{1}$, the members of a family of quarks and leptons have the following quantum numbers: 


$$
\begin{aligned}
& Q(\mathbf{3}, \mathbf{2} ; 1, w, 0)_{1 / 6} \\
& u^{c}(\overline{\mathbf{3}}, \mathbf{1} ;-1,0, x)_{-2 / 3} \\
& d^{c}(\overline{\mathbf{3}}, \mathbf{1} ;-1,0, y)_{1 / 3} \\
& L(\mathbf{1}, \mathbf{2} ; 0,1, z)_{-1 / 2} \\
& l^{c}(\mathbf{1}, \mathbf{1} ; 0,0,1)_{1}
\end{aligned}
$$

The values of the $U(1)$ charges $x, y, z, w$ will be fixed below so that they lead to the right hypercharges, shown for completeness as subscripts.

It turns out that there are two possible ways of embedding the Standard Model particle spectrum on these stacks of branes 32, which are shown pictorially in Fig. 10 The quark doublet $Q$ corresponds necessarily to a massless

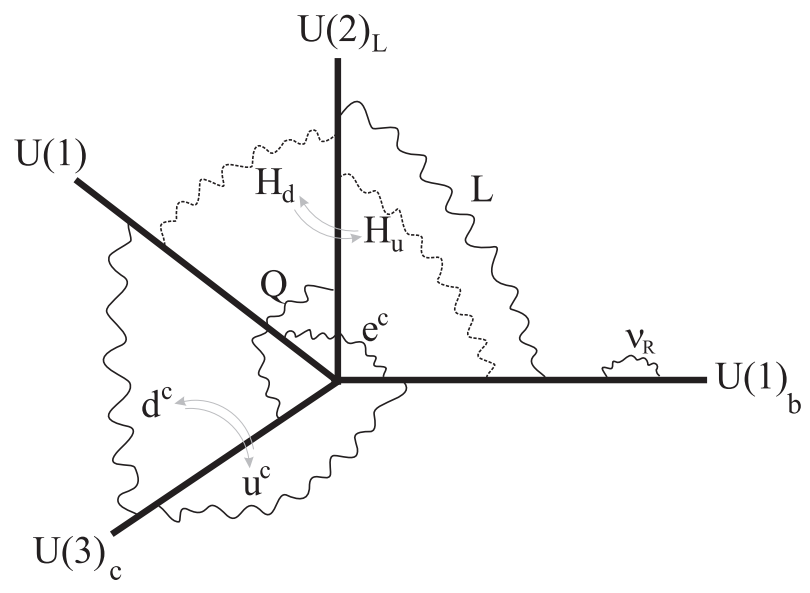

Fig. 10. A minimal Standard Model embedding on D-branes.

excitation of an open string with its two ends on the two different collections of branes (color and weak). As seen from the figure, a fourth brane stack is needed for a complete embedding, which is chosen to be a $U(1)_{b}$ extended in the bulk. This is welcome since one can accommodate right handed neutrinos as open string states on the bulk with sufficiently small Yukawa couplings suppressed by the large volume of the bulk [34. The two models are obtained by an exchange of the up and down antiquarks, $u^{c}$ and $d^{c}$, which correspond to open strings with one end on the color branes and the other either on the $U(1)$ brane, or on the $U(1)_{b}$ in the bulk. The lepton doublet $L$ arises from an open string stretched between the weak branes and $U(1)_{b}$, while the antilepton $l^{c}$ corresponds to a string with one end on the $U(1)$ brane and the other in the bulk. For completeness, we also show the two possible Higgs states $H_{u}$ and $H_{d}$ that are both necessary in order to give tree-level masses to all quarks and leptons of the heaviest generation. 
The weak hypercharge $Y$ is a linear combination of the three $U(1)$ 's:

$$
Y=Q_{1}+\frac{1}{2} Q_{2}+c_{3} Q_{3} \quad ; \quad c_{3}=-1 / 3 \text { or } 2 / 3,
$$

where $Q_{N}$ denotes the $U(1)$ generator of $U(N)$ normalized so that the fundamental representation of $S U(N)$ has unit charge. The corresponding $U(1)$ charges appearing in eq. (21) are $x=-1$ or $0, y=0$ or $1, z=-1$, and $w=1$ or -1 , for $c_{3}=-1 / 3$ or $2 / 3$, respectively. The hypercharge coupling $g_{Y}$ is given by ${ }^{4}$ :

$$
\frac{1}{g_{Y}^{2}}=\frac{2}{g_{1}^{2}}+\frac{4 c_{2}^{2}}{g_{2}^{2}}+\frac{6 c_{3}^{2}}{g_{3}^{2}}
$$

It follows that the weak angle $\sin ^{2} \theta_{W}$, is given by:

$$
\sin ^{2} \theta_{W} \equiv \frac{g_{Y}^{2}}{g_{2}^{2}+g_{Y}^{2}}=\frac{1}{2+2 g_{2}^{2} / g_{1}^{2}+6 c_{3}^{2} g_{2}^{2} / g_{3}^{2}}
$$

where $g_{N}$ is the gauge coupling of $S U(N)$ and $g_{1}=g_{2}$ or $g_{1}=g_{3}$ at the string scale. In order to compare the theoretical predictions with the experimental value of $\sin ^{2} \theta_{W}$ at $M_{s}$, we plot in Fig.[1]the corresponding curves as functions of $M_{s}$. The solid line is the experimental curve. The dashed line is the plot

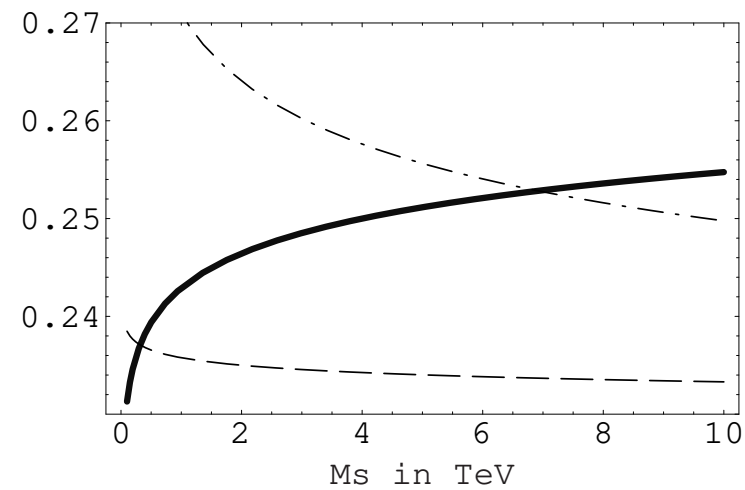

Fig. 11. The experimental value of $\sin ^{2} \theta_{W}$ (thick curve), and the theoretical predictions (24).

of the function (24) for $g_{1}=g_{2}$ with $c_{3}=-1 / 3$ while the dotted-dashed line corresponds to $g_{1}=g_{3}$ with $c_{3}=2 / 3$. The other two possibilities are not

\footnotetext{
${ }^{4}$ The gauge couplings $g_{2,3}$ are determined at the tree-level by the string coupling and other moduli, like radii of longitudinal dimensions. In higher orders, they also receive string threshold corrections.
} 
shown because they lead to a value of $M_{s}$ which is too high to protect the hierarchy. Thus, the second case, where the $U(1)$ brane is on top of the color branes, is compatible with low energy data for $M_{s} \sim 6-8 \mathrm{TeV}$ and $g_{s} \simeq 0.9$.

From Eq. (24) and Fig. 111 we find the ratio of the $S U(2)$ and $S U(3)$ gauge couplings at the string scale to be $\alpha_{2} / \alpha_{3} \sim 0.4$. This ratio can be arranged by an appropriate choice of the relevant moduli. For instance, one may choose the color and $U(1)$ branes to be $\mathrm{D} 3$ branes while the weak branes to be $\mathrm{D} 7$ branes. Then, the ratio of couplings above can be explained by choosing the volume of the four compact dimensions of the seven branes to be $V_{4}=2.5$ in string units. This being larger than one is consistent with the picture above. Moreover it predicts an interesting spectrum of KK states for the Standard model, different from the naive choices that have appeared hitherto: the only Standard Model particles that have KK descendants are the $\mathrm{W}$ bosons as well as the hypercharge gauge boson. However, since the hypercharge is a linear combination of the three $U(1)$ 's, the massive $U(1)$ KK gauge bosons do not couple to the hypercharge but to the weak doublet number.

\section{Non-compact extra dimensions and localized gravity}

There are several motivations to study localization of gravity in non-compact extra dimensions: (i) it avoids the problem of fixing the moduli associated to the size of the compactification manifold; (ii) it provides a new approach to the mass hierarchy problem; (iii) there are modifications of gravity at large distances that may have interesting observational consequences. Two types of models have been studied: warped metrics in curved space [35, and infinite size extra dimensions in flat space [36. The former, although largely inspired by stringy developments and having used many string-theoretic techniques, have not yet a clear and calculable string theory realization 37. In any case, since curved space is always difficult to handle in string theory, in the following we concentrate mainly on the latter, formulated in flat space with gravity localized on a subspace of the bulk. It turns out that these models of induced gravity have an interesting string theory realization [38 that we describe below, after presenting first a brief overview of the warped case [39].

\subsection{Warped spaces}

In these models, space-time is a slice of anti de Sitter space (AdS) in $d=5$ dimensions while our universe forms a four-dimensional (4d) flat boundary [35]. The corresponding line element is:

$$
d s^{2}=e^{-2 k|y|} \eta_{\mu \nu} d x^{\mu} d x^{\nu}+d y^{2} \quad ; \quad \Lambda=-24 M^{3} k^{2},
$$

where $M, \Lambda$ are the $5 \mathrm{~d}$ Planck mass and cosmological constant, respectively, and the parameter $k$ is the curvature of $\mathrm{AdS}_{5}$. The fifth coordinate $y$ is restricted on the interval $\left[0, \pi r_{c}\right]$. Thus, this model requires two 'branes', a UV 
and an IR, located at the two end-points of the interval, $y=0$ and $y=\pi r_{c}$, respectively. The vanishing of the $4 \mathrm{~d}$ cosmological constant requires to fine tune the two tensions: $T=-T^{\prime}=24 M^{3} k^{2}$. The $4 \mathrm{~d}$ Planck mass is given by:

$$
M_{P}^{2}=\frac{1}{k}\left(1-e^{-2 \pi k r_{c}}\right) M^{3} .
$$

Note that the IR brane can move to infinity by taking the limit $r_{c} \rightarrow \infty$, while $M_{P}$ is kept finite and thus $4 \mathrm{~d}$ gravity is always present on the brane. The reason is that the internal volume remains finite in the non-compact limit along the positive $y$ axis. As a result, gravity is kept localized on the UV brane, while the Newtonian potential gets corrections, $1 / r+1 / k^{2} r^{3}$, which are identical with those arising in the compact case of two flat extra dimensions. Using the experimental limit $k^{-1} \lesssim 0.1 \mathrm{~mm}$ and the relation (26), one finds a bound for the $5 \mathrm{~d}$ gravity scale $M \gtrsim 10^{8} \mathrm{GeV}$, corresponding to a brane tension $T \gtrsim 1 \mathrm{TeV}$. Notice that this bound is not valid in the compact case of six extra dimensions, because their size is in the fermi range and thus the $1 / r^{3}$ deviations of Newton's law are cutoff at shorter distances.

\subsection{The induced gravity model}

The DGP model and its generalizations are specified by a bulk Einstein-Hilbert (EH) term and a four-dimensional EH term [36:

$$
M^{2+n} \int_{\mathcal{M}_{4+n}} d^{4} x d^{n} y \sqrt{G} \mathcal{R}_{(4+n)}+M_{P}^{2} \int_{\mathcal{M}_{4}} d^{4} x \sqrt{g} \mathcal{R}_{(4)} ; \quad M_{P}^{2} \equiv r_{c}^{n} M^{2+n}
$$

with $M$ and $M_{P}$ the (possibly independent) respective Planck scales. The scale $M \geq 1 \mathrm{TeV}$ would be related to the short-distance scale below which UV quantum gravity or stringy effects are important. The four-dimensional metric is the restriction of the bulk metric $g_{\mu \nu}=G_{\mu \nu} \mid$ and we assume the WORLD $^{5}$ rigid, allowing the gauge $G_{i \mu} \mid=0$ with $i \geq 5$. Finally, only intrinsic curvature terms are omitted but no Gibbons-Hawking term is needed.

\section{Co-dimension one}

In the case of co-dimension one bulk $(n=1)$ and $\delta$-function localization, it is easy to see that $r_{c}$ is a crossover scale where gravity changes behavior on the WORLD. Indeed, by Fourrier transform the quadratic part of the action (27) with respect to the $4 \mathrm{~d}$ position $x$, at the WORLD position $y=0$, one obtains $M^{2+n}\left(p^{2-n}+r_{c}^{n} p^{2}\right)$, where $p$ is the $4 \mathrm{~d}$ momentum. It follows that

\footnotetext{
${ }^{5}$ We avoid calling $\mathcal{M}_{4}$ a brane because, as we will see below, gravity localizes on singularities of the internal manifold, such as orbifold fixed points. Branes with localized matter can be introduced independently of gravity localization.
} 
for distances smaller than $r_{c}$ (large momenta), the first term becomes irrelevant and the graviton propagator on the "brane" exhibits four-dimensional behavior $\left(1 / p^{2}\right)$ with Planck constant $M_{P}=M^{3} r_{c}$. On the contrary, at large distances, the first term becomes dominant and the graviton propagator acquires a five-dimensional fall-off $(1 / p)$ with Planck constant $M$. Imposing $r_{c}$ to be larger than the size of the universe, $r_{c} \gtrsim 10^{28} \mathrm{~cm}$, one finds $M \lesssim 100$ $\mathrm{MeV}$, which seems to be in conflict with experimental bounds. However, there were arguments that these bounds can be evaded, even for values of the fundamental scale $M^{-1} \sim 1 \mathrm{~mm}$ that one may need for suppressing the quantum corrections of the cosmological constant [36].

On the other hand, in the presence of non-zero brane thickness $w$, a new crossover length-scale seems to appear, $R_{c} \sim\left(w r_{c}\right)^{1 / 2}\left[40\right.$ or $r_{c}^{3 / 5} w^{2 / 5}$ [41].

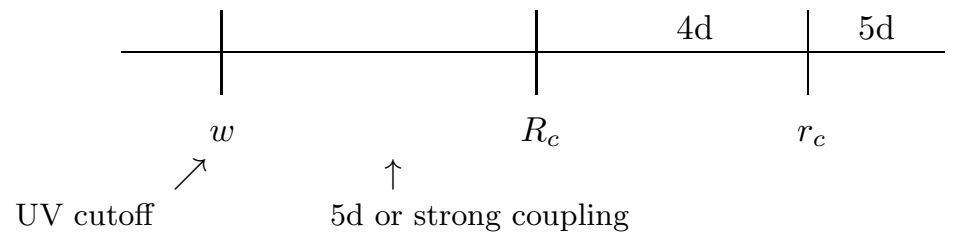

Below this scale, the theory acquires either again a five-dimensional behavior, or a strong coupling regime. For $r_{c} \sim 10^{28} \mathrm{~cm}$, the new crossover scale is of order $R_{c} \sim 10^{-4}-10 \mathrm{~m}$.

\section{Higher co-dimension}

The situation changes drastically for more than one non-compact bulk dimensions, $n>1$, due to the ultraviolet properties of the higher-dimensional theories. Indeed, from the action (27), the effective potential between two test masses in four dimensions

$$
\begin{aligned}
\int\left[d^{3} x\right] e^{-i p \cdot x} V(x) & =\frac{D(p)}{1+r_{c}^{n} p^{2} D(p)}\left[\tilde{T}_{\mu \nu} T^{\mu \nu}-\frac{1}{2+n} \tilde{T}_{\mu}^{\mu} T_{\nu}^{\nu}\right] \\
D(p) & =\int\left[d^{n} q\right] \frac{f_{w}(q)}{p^{2}+q^{2}}
\end{aligned}
$$

is a function of the bulk graviton retarded Green's function $G(x, 0 ; 0,0)=$ $\int\left[d^{4} p\right] e^{i p \cdot x} D(p)$ evaluated for two points localized on the WORLD $\left(y=y^{\prime}=0\right)$. The integral (29) is UV-divergent for $n>1$ unless there is a non-trivial brane thickness profile $f_{w}(q)$ of width $w$. If the four-dimensional WORLD has zero thickness, $f_{w}(q) \sim 1$, the bulk graviton does not have a normalizable wave function. It therefore cannot contribute to the induced potential, which always takes the form $V(p) \sim 1 / p^{2}$ and Newton's law remains four-dimensional at all distances.

For a non-zero thickness $w$, there is only one crossover length scale, $R_{c}$ : 


$$
R_{c}=w\left(\frac{r_{c}}{w}\right)^{\frac{n}{2}}
$$

above which one obtains a higher-dimensional behaviour 42 . Therefore the effective potential presents two regimes: (i) at short distances $\left(w \ll r \ll R_{c}\right)$ the gravitational interactions are mediated by the localized four-dimensional graviton and Newton's potential on the WORLD is given by $V(r) \sim 1 / r$ and, (ii) at large distances $\left(r \gg R_{c}\right)$ the modes of the bulk graviton dominate, changing the potential. Note that for $n=1$ the expressions (28) and (29) are finite and unambiguously give $V(r) \sim 1 / r$ for $r \gg r_{c}$. For a co-dimension bigger than 1, the precise behavior for large-distance interactions depends crucially on the UV completion of the theory.

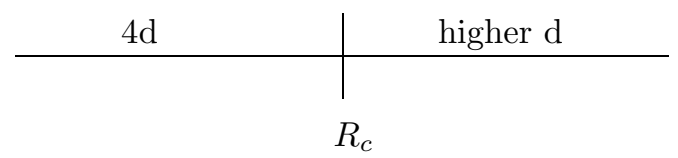

At this point we stress a fundamental difference with the finite extra dimensions scenarios. In these cases Newton's law gets higher-dimensional at distances smaller than the characteristic size of the extra dimensions. This is precisely the opposite of the case of infinite volume extra dimensions that we discuss here.

As mentioned above, for higher co-dimension, there is an interplay between UV regularization and IR behavior of the theory. Indeed, several works in the literature raised unitarity 43] and strong coupling problems 44 which depend crucially on the UV completion of the theory. A unitary UV regularization for the higher co-dimension version of the model has been proposed in 45. It would be interesting to address these questions in a precise string theory context. Actually, using for UV cutoff on the "brane" the 4d Planck length $w \sim l_{P}$, one gets for the crossover scale (30): $R_{c} \sim M^{-1}\left(M_{P} / M\right)^{n / 2}$. Putting $M \gtrsim 1 \mathrm{TeV}$ leads to $R_{c} \lesssim 10^{8(n-2)} \mathrm{cm}$. Imposing $R_{c} \gtrsim 10^{28} \mathrm{~cm}$, one then finds that the number of extra dimensions must be at least six, $n \geq 6$, which is realized nicely in string theory and provides an additional motivation for studying possible string theory realizations.

\subsection{String theory realization}

In the following, we explain how to realize the gravity induced model (27) with $n \geq 6$ as the low-energy effective action of string theory on a non-compact sixdimensional manifold $\mathcal{M}_{6} 38$. We work in the context of $\mathcal{N}=2$ supergravities in four dimensions but the mechanism for localizing gravity is independent of the number of supersymmetries. Of course for $\mathcal{N} \geq 3$ supersymmetries, there is no localization. We also start with the compact case and take the 
decompactification limit. The localized properties are then encoded in the different volume dependences.

In string perturbation, corrections to the four-dimensional Planck mass are in general very restrictive. In the heterotic string, they vanish to all orders in perturbation theory [46; in type I theory, there are moduli-dependent corrections generated by open strings [47, but they vanish when the manifold $\mathcal{M}_{6}$ is decompactified; in type II theories, they are constant, independent of the moduli of the manifold $\mathcal{M}_{6}$, and receive contributions only from tree and one-loop levels that we describe below (at least for supersymmetric backgrounds) 38, 48. Finally, in the context of M-theory, one obtains a similar localized action of gravity kinetic terms in five dimensions, corresponding to the strong coupling limit of type IIA string 38 .

The origin of the two EH terms in (27) can be traced back to the perturbative corrections to the eight-derivative effective action of type II strings in ten dimensions. These corrections include the tree-level and one-loop terms given by: ${ }^{6}$

$$
\begin{aligned}
\frac{1}{l_{s}^{8}} & \int_{M_{10}} \frac{1}{g_{s}^{2}} \mathcal{R}_{(10)}+\frac{1}{l_{s}^{2}} \int_{M_{10}}\left(\frac{2 \zeta(3)}{g_{s}^{2}}+4 \zeta(2)\right) t_{8} t_{8} R^{4} \\
& -\frac{1}{l_{s}^{2}} \int_{M_{10}}\left(\frac{2 \zeta(3)}{g_{s}^{2}} \mp 4 \zeta(2)\right) R \wedge R \wedge R \wedge R \wedge e \wedge e+\cdots
\end{aligned}
$$

where $\phi$ is the dilaton field determining the string coupling $g_{s}=e^{\langle\phi\rangle}$, and the \pm sign corresponds to the type IIA/B theory.

On a direct product space-time $\mathcal{M}_{6} \times \mathbb{R}^{4}$, the $t_{8} t_{8} R^{4}$ contribute in four dimensions to $R^{2}$ and $R^{4}$ terms [48. At the level of zero modes, the second $R^{4}$ term in (31) splits as:

$$
\int_{M_{6}} R \wedge R \wedge R \times \int_{M_{4}} \mathcal{R}_{(4)}=\chi \int_{M_{4}} \mathcal{R}_{(4)},
$$

where $\chi$ is the Euler number of the $M_{6}$ compactification manifold. We thus obtain the action terms:

$$
\frac{1}{l_{s}^{8}} \int_{M_{4} \times M_{6}} \frac{1}{g_{s}^{2}} \mathcal{R}_{(10)}+\frac{\chi}{l_{s}^{2}} \int_{M_{4}}\left(-\frac{2 \zeta(3)}{g_{s}^{2}} \pm 4 \zeta(2)\right) \mathcal{R}_{(4)},
$$

which gives the expressions for the Planck masses $M$ and $M_{p}$ :

$$
M^{2} \sim M_{s}^{2} / g_{s}^{1 / 2} \quad ; \quad M_{P}^{2} \sim \chi\left(\frac{c_{0}}{g_{s}^{2}}+c_{1}\right) M_{s}^{2},
$$

with $c_{0}=-2 \zeta(3)$ and $c_{1}= \pm 4 \zeta(2)= \pm 2 \pi^{2} / 3$.

\footnotetext{
${ }^{6}$ The rank-eight tensor $t_{8}$ is defined as $t_{8} M^{4} \equiv-6\left(\operatorname{tr} M^{2}\right)^{2}+24 \operatorname{tr} M^{4}$. See [49] for more details.
} 
It is interesting that the appearance of the induced $4 \mathrm{~d}$ localized term preserves $\mathcal{N}=2$ supersymmetry and is independent of the localization mechanism of matter fields (for instance on D-branes). Localization requires the internal space $M_{6}$ to have a non-zero Euler characteristic $\chi \neq 0$. Actually, in type IIA/B compactified on a Calabi-Yau manifold, $\chi$ counts the difference between the numbers of $\mathcal{N}=2$ vector multiplets and hypermultiplets: $\chi= \pm 4\left(n_{V}-n_{H}\right)$ (where the graviton multiplet counts as one vector). Moreover, in the non-compact limit, the Euler number can in general split in different singular points of the internal space, $\chi=\sum_{I} \chi_{I}$, giving rise to different localized terms at various points $y_{I}$ of the internal space. A number of conclusions (confirmed by string calculations in 38]) can be reached by looking closely at (33):

$\triangleright M_{p} \gg M$ requires a large non-zero Euler characteristic for $M_{6}$, and/or a weak string coupling constant $g_{s} \rightarrow 0$.

$\triangleright$ Since $\chi$ is a topological invariant the localized $\mathcal{R}_{(4)}$ term coming from the closed string sector is universal, independent of the background geometry and dependent only on the internal topology ${ }^{7}$. It is a matter of simple inspection to see that if one wants to have a localized EH term in less than ten dimensions, namely something linear in curvature, with non-compact internal space in all directions, the only possible dimension is four (or five in the strong coupling M-theory limit).

$\triangleright$ In order to find the width $w$ of the localized term, one has to do a separate analysis. On general grounds, using dimensional analysis in the limit $M_{P} \rightarrow \infty$, one expects the effective width to vanish as a power of $l_{P} \equiv M_{P}^{-1}$ : $w \sim l_{P}^{\nu} / l_{s}^{\nu-1}$ with $\nu>0$. The computation of $\nu$ for a general Calabi-Yau space, besides its technical difficulty, presents an additional important complication: from the expression (34), $l_{P} \sim g_{s} l_{s}$ in the weak coupling limit. Thus, $w$ vanishes in perturbation theory and one has to perform a non-perturbative analysis to extract its behavior. Alternatively, one can examine the case of orbifolds. In this limit, $c_{0}=0, l_{P} \sim l_{s}$, and the hierarchy $M_{P}>M$ is achieved only in the limit of large $\chi$.

The one-loop graviton amplitude for the supersymmetric orbifold $T^{6} / \mathbb{Z}_{N}$, takes the form of a sum of quasi-localized contributions at the positions of the fixed points $x_{f}$ of the orbifold [38]:

$$
\left\langle V_{g}^{3}\right\rangle \sim \frac{1}{N} \sum_{(h, g)} \sum_{x_{f}} \int_{\mathcal{F}} \frac{d^{2} \tau}{\tau_{2}^{2}} \int \prod_{i=1}^{3} \frac{d^{2} z_{i}}{\tau_{2}} \frac{1}{F_{(h, g)}\left(\tau, z_{i}\right)^{3}} e^{-\frac{\left(y-x_{f}\right)^{2}}{\alpha^{\prime} F(h, g)}\left(\tau, z_{i}\right)},
$$

where $(h, g)$ denote the orbifold twists and $\tau=\tau_{1}+i \tau_{2}$ is the complex modulus of the world-sheet torus, integrated over its fundamental domain $\mathcal{F}$. The above

\footnotetext{
${ }^{7}$ Field theory computations of [50] show that the Planck mass renormalization depends on the UV behavior of the matter fields coupling to the external metric. But, even in the supersymmetric case, the corrections are not obviously given by an index.
} 
expression (35) gives the three-point amplitude involving three $4 \mathrm{~d}$ gravitons on-shell. Focusing on one particular fixed point $x_{f}=0$ and sending the radii to infinity, we obtain the effective action for the quasi-localized EH term

$$
\chi \int d^{4} x d^{6} y \sqrt{g} f_{w}(y) \mathcal{R}_{(4)}
$$

with a width given by the four-dimensional induced Planck mass

$$
w \simeq l_{P}=l_{s} \chi^{-1 / 2},
$$

and the power $\nu=1$.

\section{Summary of the results}

Using $w \sim l_{P}$ and the relations (34) in the weak coupling limit (with $c_{0} \neq 0$ ), the crossover radius of eq. (30) is given by the string parameters $(n=6)$

$$
R_{c}=\frac{r_{c}^{3}}{w^{2}} \sim g_{s} \frac{l_{s}^{4}}{l_{P}^{3}} \simeq g_{s} \times 10^{32} \mathrm{~cm},
$$

for $M_{s} \simeq 1 \mathrm{TeV}$. Because $R_{c}$ has to be of cosmological size, the string coupling can be relatively small, and the Euler number $|\chi| \simeq g_{s}^{2} l_{P} \sim g_{s}^{2} \times 10^{32}$ must be very large. The hierarchy is obtained mainly thanks to the large value of $\chi$, so that lowering the bound on $R_{c}$ lowers the value of $\chi$. Our actual knowledge of gravity at very large distances indicates $\left[51\right.$ that $R_{c}$ should be of the order of the Hubble radius $R_{c} \simeq 10^{28} \mathrm{~cm}$, which implies $g_{s} \geq 10^{-4}$ and $|\chi| \gtrsim 10^{24}$. A large Euler number implies only a large number of closed string massless particles with no a-priori constraint on the observable gauge and matter sectors, which can be introduced for instance on D3-branes placed at the position where gravity localization occurs. All these particles are localized at the orbifold fixed points (or where the Euler number is concentrated in the general case), and should have sufficiently suppressed gravitational-type couplings, so that their presence with such a huge multiplicity does not contradict observations. Note that these results depend crucially on the scaling of the width $w$ in terms of the Planck length: $w \sim l_{P}^{\nu}$, implying $R_{c} \sim 1 / l_{P}^{2 \nu+1}$ in string units. If there are models with $\nu>1$, the required value of $\chi$ will be much lower, becoming $\mathcal{O}(1)$ for $\nu \geq 3 / 2$. In this case, the hierarchy could be determined by tuning the string coupling to infinitesimal values, $g_{s} \sim 10^{-16}$.

The explicit string realization of localized induced gravity models offers a consistent framework that allows to address a certain number of interesting physics problems. In particular, the effective UV cutoff and the study of the gravity force among matter sources localized on D-branes. It would be also interesting to perform explicit model building and study in detail the phenomenological consequences of these models and compare to other realizations of $\mathrm{TeV}$ strings with compact dimensions. 


\section{Acknowledgments}

This work was supported in part by the European Commission under the RTN contract MRTN-CT-2004-503369, and in part by the INTAS contract 03-51-6346.

\section{References}

1. C. Angelantonj and A. Sagnotti, Phys. Rept. 371, 1 (2002) [Erratum-ibid. 376, 339 (2003)] arXiv:hep-th/0204089.

2. I. Antoniadis, Phys. Lett. B 246, 377 (1990).

3. C. D. Hoyle, D. J. Kapner, B. R. Heckel, E. G. Adelberger, J. H. Gundlach, U. Schmidt and H. E. Swanson, Phys. Rev. D 70, 042004 (2004).

4. N. Arkani-Hamed, S. Dimopoulos and G. R. Dvali, Phys. Lett. B 429, 263 (1998) arXiv:hep-ph/9803315; I. Antoniadis, N. Arkani-Hamed, S. Dimopoulos and G. R. Dvali, Phys. Lett. B 436, 257 (1998) arXiv:hep-ph/9804398.

5. For a review see e.g. I. Antoniadis, Prepared for NATO Advanced Study Institute and EC Summer School on Progress in String, Field and Particle Theory, Cargese, Corsica, France (2002); and references therein.

6. J. D. Lykken, Phys. Rev. D 54, 3693 (1996) arXiv:hep-th/9603133.

7. J. C. Long and J. C. Price, Comptes Rendus Physique 4, 337 (2003); R. S. Decca, D. Lopez, H. B. Chan, E. Fischbach, D. E. Krause and C. R. Jamell, Phys. Rev. Lett. 94, 240401 (2005); S. J. Smullin, A. A. Geraci, D. M. Weld, J. Chiaverini, S. Holmes and A. Kapitulnik, arXiv:hep-ph/0508204 H. Abele, S. Haeßler and A. Westphal, in 271th WE-Heraeus-Seminar, Bad Honnef (2002).

8. I. Antoniadis and K. Benakli, Phys. Lett. B 326, 69 (1994).

9. I. Antoniadis, K. Benakli and M. Quirós, Phys. Lett. B 331, 313 (1994) and Phys. Lett. B 460, 176 (1999); P. Nath, Y. Yamada and M. Yamaguchi, Phys. Lett. B 466, 100 (1999) T. G. Rizzo and J. D. Wells, Phys. Rev. D 61, 016007 (2000); T. G. Rizzo, Phys. Rev. D 61, 055005 (2000); A. De Rujula, A. Donini, M. B. Gavela and S. Rigolin, Phys. Lett. B 482, 195 (2000);

10. E. Accomando, I. Antoniadis and K. Benakli, Nucl. Phys. B 579, 3 (2000).

11. I. Antoniadis, K. Benakli and A. Laugier, JHEP 0105, 044 (2001).

12. P. Nath and M. Yamaguchi, Phys. Rev. D 60, 116004 (1999); Phys. Rev. D 60, 116006 (1999); M. Masip and A. Pomarol, Phys. Rev. D 60, 096005 (1999); W. J. Marciano, Phys. Rev. D 60, 093006 (1999); A. Strumia, Phys. Lett. B 466, 107 (1999); R. Casalbuoni, S. De Curtis, D. Dominici and R. Gatto, Phys. Lett. B 462, 48 (1999); C. D. Carone, Phys. Rev. D 61, 015008 (2000); A. Delgado, A. Pomarol and M. Quirós, JHEP 1, 30 (2000).

13. G. Servant and T. M. P. Tait, Nucl. Phys. B 650, 391 (2003).

14. G. F. Giudice, R. Rattazzi and J. D. Wells, Nucl. Phys. B 544, 3 (1999); E. A. Mirabelli, M. Perelstein and M. E. Peskin, Phys. Rev. Lett. 82, 2236 (1999); T. Han, J. D. Lykken and R. Zhang, Phys. Rev. D 59, 105006 (1999); K. Cheung and W.-Y. Keung, Phys. Rev. D 60, 112003 (1999); C. Balázs et al., Phys. Rev. Lett. 83, 2112 (1999); L3 Collaboration (M. Acciarri et al.), Phys. Lett. B 464, 135 (1999) and 470, 281 (1999): J. L. Hewett, Phys. Rev. Lett. 82, 4765 (1999). 
15. N. Arkani-Hamed, S. Dimopoulos and G. Dvali, Phys. Rev. D 59, 086004 (1999).

16. S. Cullen and M. Perelstein, Phys. Rev. Lett. 83, 268 (1999); V. Barger, T. Han, C. Kao and R. J. Zhang, Phys. Lett. B 461, 34 (1999).

17. K. Benakli and S. Davidson, Phys. Rev. D 60, 025004 (1999); L. J. Hall and D. Smith, Phys. Rev. D 60, 085008 (1999).

18. S. Cullen, M. Perelstein and M. E. Peskin, Phys. Rev. D 62, 055012 (2000); D. Bourilkov, Phys. Rev. D 62, 076005 (2000); L3 Collaboration (M. Acciarri et al.), Phys. Lett. B 489, 81 (2000).

19. S. B. Giddings and S. Thomas, Phys. Rev. D 65, 056010 (2002); S. Dimopoulos and G. Landsberg, Phys. Rev. Lett. 87, 161602 (2001).

20. I. Antoniadis, C. Bachas, Phys. Lett. B 450, 83 (1999).

21. I. Antoniadis, K. Benakli, A. Laugier and T. Maillard, Nucl. Phys. B 662, 40 (2003) arXiv:hep-ph/0211409

22. I. Antoniadis, S. Dimopoulos and G. Dvali, Nucl. Phys. B 516, 70 (1998); S. Ferrara, C. Kounnas and F. Zwirner, Nucl. Phys. B 429, 589 (1994).

23. T. R. Taylor and G. Veneziano, Phys. Lett. B 213, 450 (1988).

24. I. Antoniadis, E. Kiritsis and J. Rizos, Nucl. Phys. B 637, 92 (2002).

25. D. V. Volkov and V. P. Akulov, JETP Lett. 16, 438 (1972) and Phys. Lett. B 46, 109 (1973).

26. A. Brignole, F. Feruglio and F. Zwirner, JHEP 9711, 001 (1997); T. E. Clark, T. Lee, S. T. Love and G. Wu, Phys. Rev. D 57, 5912 (1998); M. A. Luty and E. Ponton, Phys. Rev. D 57, 4167 (1998); I. Antoniadis, K. Benakli and A. Laugier, Nucl. Phys. B 631, 3 (2002).

27. I. Antoniadis and M. Tuckmantel, Nucl. Phys. B 697, 3 (2004).

28. I. Antoniadis, M. Tuckmantel and F. Zwirner, Nucl. Phys. B 707, 215 (2005) arXiv:hep-ph/0410165.

29. I. Antoniadis, K. Benakli and M. Quirós, Nucl. Phys. B 583, 35 (2000).

30. I. Antoniadis, K. Benakli and M. Quiros, New Jour. Phys. 3, 20 (2001).

31. I. Antoniadis, C. Muñoz and M. Quirós, Nucl. Phys. B 397, 515 (1993); I. Antoniadis and M. Quirós, Phys. Lett. B 392, 61 (1997); A. Pomarol and M. Quirós, Phys. Lett. B 438, 225 (1998); I. Antoniadis, S. Dimopoulos, A. Pomarol and M. Quirós, Nucl. Phys. B 544, 503 (1999); A. Delgado, A. Pomarol and M. Quirós, Phys. Rev. D 60, 095008 (1999); R. Barbieri, L. J. Hall and Y. Nomura, Phys. Rev. D 63, 105007 (2001).

32. I. Antoniadis, E. Kiritsis and T. N. Tomaras, Phys. Lett. B 486, 186 (2000); I. Antoniadis, E. Kiritsis, J. Rizos and T. N. Tomaras, Nucl. Phys. B 660, 81 (2003).

33. G. Shiu and S.-H. H. Tye, Phys. Rev. D 58, 106007 (1998); Z. Kakushadze and S.-H. H. Tye, Nucl. Phys. B 548, 180 (1999); L. E. Ibáñez, C. Muñoz and S. Rigolin, Nucl. Phys. B 553, 43 (1999).

34. K. R. Dienes, E. Dudas and T. Gherghetta, Nucl. Phys. B 557, 25 (1999) arXiv:hep-ph/9811428; N. Arkani-Hamed, S. Dimopoulos, G. R. Dvali and J. March-Russell, Phys. Rev. D 65, 024032 (2002) arXiv:hep-ph/9811448; G. R. Dvali and A. Y. Smirnov, Nucl. Phys. B 563, 63 (1999).

35. L. Randall and R. Sundrum, Phys. Rev. Lett. 83, 4690 (1999) and Phys. Rev. Lett. 83, 3370 (1999).

36. G. R. Dvali, G. Gabadadze and M. Porrati, Phys. Lett. B 485, 208 (2000).

37. H. Verlinde, Nucl. Phys. B 580, 264 (2000); S. B. Giddings, S. Kachru and J. Polchinski, Phys. Rev. D 66, 106006 (2002). 
38. I. Antoniadis, R. Minasian and P. Vanhove, Nucl. Phys. B 648, 69 (2003) arXiv:hep-th/0209030.

39. For a recent review see e.g. R. Maartens, Living Rev. Rel. 7, 7 (2004) arXiv:gr-qc/0312059; same proceedings and references therein.

40. E. Kiritsis, N. Tetradis and T. N. Tomaras, JHEP 0108, 012 (2001).

41. M. A. Luty, M. Porrati and R. Rattazzi, arXiv:hep-th/0303116

42. G. R. Dvali and G. Gabadadze, Phys. Rev. D 63, 065007 (2001); G. R. Dvali, G. Gabadadze, M. Kolanovic and F. Nitti, Phys. Rev. D 64, 084004 (2001).

43. S. L. Dubovsky and V. A. Rubakov, Phys. Rev. D 67, 104014 (2003) arXiv:hep-th/0212222.

44. V. A. Rubakov, arXiv:hep-th/0303125

45. M. Kolanovic, M. Porrati and J. W. Rombouts, Phys. Rev. D 68, 064018 (2003) arXiv:hep-th/0304148.

46. I. Antoniadis, E. Gava and K. S. Narain, Phys. Lett. B 283, 209 (1992).

47. I. Antoniadis, C. Bachas, C. Fabre, H. Partouche and T. R. Taylor, Nucl. Phys. B 489, 160 (1997); I. Antoniadis, H. Partouche and T. R. Taylor, Nucl. Phys. B 499, 29 (1997).

48. I. Antoniadis, S. Ferrara, R. Minasian and K. S. Narain, Nucl. Phys. B 507, 571 (1997).

49. K. Peeters, P. Vanhove and A. Westerberg, Class. Quant. Grav. 18, 843 (2001).

50. S. L. Adler, Rev. Mod. Phys. 54, 729 (1982) [Erratum-ibid. 55 (1983) 837].

51. A. Lue and G. Starkman, Phys. Rev. D 67, 064002 (2003). 